\title{
NLRP3 Inflammasome Activation Attenuates Neuronal Apoptosis by Upregulating Autophagy through AMPK/Beclin-1 Pathway after Intracerebral Hemorrhage with Ventricular Extension in Rats
}

\section{Zhaoqi Zhang}

Third Military Medical University Southwest Hospital

\section{Peiwen Guo}

Third Military Medical University Southwest Hospital

Zhengcai Jia

Third Military Medical University Southwest Hospital

\section{Tunan Chen}

Third Military Medical University: Army Medical University

Hua Feng ( $\square$ fenghua8888@vip.163.com )

Third military medical university https://orcid.org/0000-0003-4489-9217

\section{Research}

Keywords: NLRP3 inflammasome, autophagy, apoptosis, microglia/macrophage, neurons, IL-1 $\beta$

Posted Date: February 17th, 2021

DOI: https://doi.org/10.21203/rs.3.rs-203453/v1

License: (c) (1) This work is licensed under a Creative Commons Attribution 4.0 International License. Read Full License 


\section{Abstract}

\section{Background}

In brain, NLRP3 inflammasome, mainly derived from macrophage/microglia, is involved in proinflammatory and neurodeficits after hemorrhage, and autophagy is vital for neuronal homeostasis and functions. Accumulating evidence suggested that NLRP3 inflammasome and autophagy played an important role in intracerebral hemorrhage $(\mathrm{ICH})$. Thus, this study was designed to further explore the pathogenesis of neurodeficits after in posthemorrhagic hydrocephalus.

Methods

Autologous blood injection model was induced to mimic ICH with ventricular extension (ICH-IVH) in Sprague-Dawley rats. To elucidate the underlying mechanism, the NLRP3 inflammasome inhibitor MCC950 was administered abdominally at $1 \mathrm{~h}$ after ICH-IVH. Magnetic resonance imaging, neurobehavioral tests, immunofluorescence, western blotting, Fluoro-Jade C- staining, Tunel staining, and Quantitative RNA Sequencing were performed.

Results

In the acute phase of ICH-IVH, both the expression of NLRP3 inflammasome and the autophagy of neurons were upregulated. The activated NLRP3 in macrophage/microglia promoted the release of IL-1 $\beta$ to extracellular, which contributed to excessive autophagy, leading to neurons apoptosis both in vivo and in vitro. AMPK/Beclin-1 pathway played an important role in NLRP3-related neurons autophagy. Using MCC950(NLRP3 inflammasome specific inhibitor) treatment after ICH-IVH significantly reduced ventricles dilation, improved neurofunction, down-regulated the release of $\mathrm{IL}-1 \beta$, and alleviated neuroinflammation and excessive autophagy.

\section{Conclusions}

Our finding demonstrated that NLRP3 inflammasome activated in microglia/macrophage aggravated neurological outcomes and neuronal apoptosis by upregulating autophagy after $\mathrm{ICH}-\mathrm{IVH}$, which was partly mediated by the AMPK/Beclin-1 pathway. Therefore, inhibiting the activation of NLRP3 may be a potential therapeutic strategy for the neurodeficits of ICH-IVH patients.

\section{Background}

Intracerebral hemorrhage $(\mathrm{ICH})$ has a high morbidity and mortality and is associated with severe longterm disability[1, 2], however, there is still no effective treatment so far[3]. About $40 \%$ of ICH break into the ventricle (ICH-IVH), and more than half of ICH-IVH patients will develop into varying degrees of hydrocephalus, which make the unfavorable outcome even worse $[2,4,5]$. Although there are many hypotheses about the pathogenesis of hydrocephalus after ICH-IVH, such as blood-clot blockage, barrier 
impairment, inflammation and blood components[6], due to the rare experimental verification, the exact pathogenesis remains unclear.

Once ICH-IVH occurred, the resident microglia/macrophage and recruited peripheral leukocytes were soon activated to release proinflammation cytokines, causing neuroinflammation and brain injury at acute phase[7-9]. The NLRP3 inflammasome, highly expressed in microglia/macrophage, has been proved to participate in a variety of pathological process such as psychiatric and neurodegenerative disorders[10, 11]. By activation of NLRP3 inflammasome can induce cytokines, such as IL-1 $\beta$, which was a new cardiovascular risk biomarker[12]. Accumulating evidences that NLRP3 inflammasome could relieve neuroinflammation and reduce cell death in early brain injury[13,14] make the NLRP3 inflammasomeinduced anti-inflammatory treatment be a potential strategy.

Autophagy is an evolutionarily conserved intracellular process to maintain cellular homeostasis by the phagosome and lysosomal pathways[15]. Abnormal autophagy has been found to play an important role in the pathogenic process of a variety of neurodegenerative diseases[16-18], such as Parkinson disease $(P D)$ and Alzheimer disease (AD) $[19,20]$. What's more, recent studies have revealed that autophagy is upregulated under some restress conditions in neurons[21] and excessive autophagy can lead to neurons apoptosis after ischemia[22]. However, there are few reports about autophagy dysfunction in acute phase of $\mathrm{ICH}$.

Herein, we hypothesized that autophagy might have a role in ICH-IVH. Since inflammation and autophagy were closely related, we further explored the relationship between NLRP3 inflammasome (an important molecular regulator in inflammation) activation in microglia/macrophage and autophagy in neurons after $\mathrm{ICH}-\mathrm{IVH}$. To address this aim, a rat model of $\mathrm{ICH}$ with ventricular extension and $\mathrm{PC} 12$ cells were used.

\section{Methods}

\section{Animals}

All experimental procedures were approved by the institutional animal care and use committee of the third military university and were performed according to the guide for the care and use of laboratory animals of the national institutes of health and reported in compliance with the ARRIVE (animal research: reporting of in vivo experiments) guidelines. A total of 261 male rats (weight 220-250g) were housed in a temperature and humidity controlled room under a standard 12-h light/dark cycle for a minimum of 3 days before ICH-IVH induction and were provided free access to food and water.

\section{ICH-IVH Model and Groups}

$\mathrm{ICH}-\mathrm{IVH}$ was induced by autologous arterial blood injection into the right peri-lateral ventricles as previously described[23]. Briefly, rats were anesthetized with pentobarbital $(40 \mathrm{mg} / \mathrm{kg}, \mathrm{IP})$. The right femoral artery was catheterized as a source of blood sample. A cranial burr hole $(1 \mathrm{~mm})$ was drilled after rats were positioned in a stereotaxic frame. Aliquots of $200 \mu$ l nonheparinized arterial blood was infused 
into the right caudate nucleus (coordinates: $0.2 \mathrm{~mm}$ posterior, $2.2 \mathrm{~mm}$ lateral, and $5.0 \mathrm{~mm}$ ventral to the bregma) at a rate of $14 \mu \mathrm{l} / \mathrm{min}$ using a microinfusion pump. The burr hole was sealed with bone wax and skin incision was closed with sutures after the needle was removed.

Rats were randomly divided into the following 3 group: sham, ICH-IVH, and MCC950. The

sham group had only a needle insertion. MCC950 group received MCC950(10mg/kg, IP; MCE, USA) at 1 hour after ICH-IVH model. ICH-IVH group was given an equal volume of phosphate-buffered saline (PBS) at the same time.

\section{Cell Culture and Treatment}

PC12 neurons cells of rats (ScienCell, USA) were used for in vitro study. PC12 cells were cultured in Dulbecco's Modified Eagle Medium (DMEM) supplemented with $10 \%(\mathrm{v} / \mathrm{v})$ fetal bovine serum (FBS) and $1 \%$ penicillin/streptomycin at $37^{\circ} \mathrm{C}$ in a humidified atmosphere of $5 \% \mathrm{CO}_{2}$. After the completion of cell processing, IL-1 $\beta$ (Novoprotein, China) was added into DMEM and diluted to different concentrations $(0 \mu \mathrm{g} / \mathrm{L}, 10 \mu \mathrm{g} / \mathrm{L}, 20 \mu \mathrm{g} / \mathrm{L})$. PC12 cells were then treated with different dosages of $\mathrm{L}-1 \beta$ for $24 \mathrm{~h}$.

\section{Apoptosis Assay}

Apoptosis was detected using ANNEXIN V-FITC/PI cell apoptosis detection kit (CST, USA). PC12 cells from different groups were digested with trypsin but without EDTA, resuspended in the blinding buffer, and stained with Annexin V-FITC for $15 \mathrm{~min}$ and PI for $5 \mathrm{~min}$. The results were analyzed by flow cytometry (Canto2, BD, USA).

\section{Immunofluorescence Staining}

Under deep anesthesia, rats were sacrificed by transcardial perfusion with $100 \mathrm{ml}$ normal saline followed by $50 \mathrm{ml} 4 \%$ neutral buffered Paraformaldehyde. Brains were fixed in $4 \%$ neutral buffered paraformaldehyde for $24 \mathrm{~h}$ at $4{ }^{\circ} \mathrm{C}$ followed by $25 \%$ and $30 \%$ sucrose solution until brains were dehydrated fully. Then brains were cut into $10 \mu \mathrm{m}$ thick coronal sections using a cryostat (LM3050S, Leica, Germany) after being frozen at $-80^{\circ} \mathrm{C}$. Slides were washed with $0.01 \mathrm{M}$ of PBS 3 times for 10 min and then incubated in $0.3 \%$ Triton X-100 for $30 \mathrm{~min}$ at room temperature. After being blocked with $5 \% \mathrm{BSA}$ for $1 \mathrm{~h}$ at room temperature, the sections were incubated with primary antibody at $4{ }^{\circ} \mathrm{C}$ overnight as follows: anti-caspase1 (1:200; NOVUS; USA), anti-IL1 $\beta$ (1:400; GeneTex, USA), anti-lba1 (1:200; Genetex, USA), anti-CD68 (1:200; abcam, USA), anti-NLRP3 (1:200; abcam, USA), anti-LC3B (1:100; ZEN-BIO; China), anti-p62 (1:100; ZEN-BIO; China), and anti-NeuN (1:200; abcam, USA). Then, the sections were washed with $0.01 \mathrm{M}$ PBS and incubated with appropriate fluorescence-conjugated secondary antibodies (1:400; Invitrogen, USA) for $2 \mathrm{~h}$ at room temperature. The slides were observed and photographed under a fluorescence microscope (LSM880; ZEISS, Germany).

\section{Western Blotting}


The subventricular zone (SVZ) tissue was separated and homogenized to collect the protein samples. Equal amounts of protein samples (20ug) were loaded on SDS-PAGE gels, electrophoresed, and transferred onto a polyvinylidene difluoride membrane. The membrane was blocked and incubated overnight at $4^{\circ} \mathrm{C}$ with the following primary antibodies: anti-NLRP3 (1:1000; abcam, USA), anti-caspase1 (1:1000; NOVUS, USA), anti-IL1 $\beta$ (1:1000; GeneTex, USA), anti-LC3B (1:1000; ZEN-BIO, China), anti-p62 (1:1000; ZEN-BIO, China), anti-pAMPK (1:1000; CST, USA), anti-AMPK (1:1000; CST, USA), anti-ULK1 (1:1000; CST, USA), anti-Beclin-1 (1:1000; CST, USA), and anti- $\beta$-actin (1:1000; CST, USA). Appropriate secondary antibodies $(1: 3000, C S T ; 1: 5000$, abcam) were selected to incubate with the membrane for $1 \mathrm{~h}$ at room temperature. The bands were probed with an ECL Plus chemiluminescence regent Kit (ThermoFisher, USA) and visualized with the image system (Bio-Rad, USA). Relative density of the protein immunoblot images were analyzed by Image $\mathrm{J}$ software ( $\mathrm{NIH}, \mathrm{USA})$.

\section{TUNEL Staining}

On day 3 after ICH-IVH, the brains were sampled for TUNEL staining using Apoptosis Detection Kit (Roche, USA) according to the manufacturer's instructions. The number of TUNEL-positive cells in the SVZ was counted using Image J software ( $\mathrm{NIH}, \mathrm{USA}$ ). Six sections per brain were used for counting. Data were expressed as the number of TUNEL-positive neurons cells $/ \mathrm{mm}^{2}$ in SVZ.

\section{Fluoro-Jade C Staining}

Neurons degeneration were evaluated by Fluoro-Jade C(FJC) staining as previously reported[?]. The FJC Ready-to- Dilute Staining Kit (Biosensis Inc., Thebarton, SA, Australia) was used. Six continuous pictures of SVZ were photographed under a fluorescence microscope and the average number of FJC-positive cells was calculated as cells $/ \mathrm{mm}^{2}$ by Image $\mathrm{J}$ software.

\section{Cell Counting}

Cell counting was performed on brain coronal sections. Three high-power images ( $\times 40$ magnification) were taken in SVZ using a digital camera. Interested positive cells were counted from 4 areas in each brain section by two researchers in a blinded manner.

\section{Neurobehavior Assessment}

On day 3, 7, and 14 after ICH-IVH, the modified Neurological Severity Score (mNSS) and corner turn were used to evaluate the motor, sensory, and balance functions of animals as previously described[24]. The mNSS was divided into 3 levels based on the range of scores: 1 to 6 indicated mild injury, 7 to 12 indicated moderate injury, and 13 to 18 indicated severe injury. In the corner turn test, each rat was allowed to proceed into a corner (the angle of $30^{\circ}$ ) for 10 times with at least 30 s intervals between every trial. The rats need turn to right or left, and the percentage of right turns was calculated to assess the neurofunction. 
Rats were anesthetized with $2 \%$ isoflurane/air mixture throughout MRI examination. The MRI scans were performed in a 7.0-T Varian MR scanner (Bruker, USA) with a T2 fast spin-echo sequence using a view field of $35 \mathrm{~mm} \times 35 \mathrm{~mm}$ and 17 coronal slices (1.0mm thickness). Volumes were calculated as previously described[25]. Bilateral ventricles were outlined, and the areas were measured using Image $\mathrm{J}(\mathrm{NIH}, \mathrm{USA})$. The volumes were assessed by calculating the areas of all slices and multiplying by the section thickness. All image analyses were performed by two observers in blinded manner.

\section{Quantitative RNA Sequencing}

Rats were euthanized on day 3 after ICH-IVH. RNA-Seq experiments were performed according to manufacturer's protocol, and data were analyzed by LC Biotech. Briefly, total RNA was extracted from the SVZ tissue using TRIzol reagent, and the quantified and purified total RNA were used to reversetranscribed to generate CDNAs, which were used to synthesize U-labeled second-stranded DNAs. The ligated products were amplified with PCR, and the average inset size for the final cDNA library was $300 \mathrm{bp}$ (50 bp). The expression levels of all transcripts were evaluated by calculating the fragments per kilobase per million reads. The threshold of significantly differential expression was set to $p<0.05$ and $\| \log _{2}$ (fold change) $\mid \geq 1$. The Gene Orthology (GO) and Kyoto Encyclopedia of Genes and Genomes(KEEG) database were used to explore the biological pathways.

\section{Statistical Analysis}

All data were presented as mean \pm SD. Data were analyzed by investigators blinded to experimental treatments. All analyses were performed using GraphPad Prism 8 (GraphPad software). We determined each sample size by power analysis using a significance level of $a=0.05$ with $80 \%$ power to detect statistical differences. Statistical evaluation of the data was performed by analysis of variance (ANOVA), followed by Tukey multiple-comparison post hoc analysis. Statistical significance was defined as $p<0.05$.

\section{Results}

\section{Ventricular Dilation and Neurodeficits after ICH-IVH}

Compared with sham group, ICH-IVH led to obvious ventricles extension by time prolonged (sham vs ICH$\mathrm{IVH}, \mathrm{p}<0.01$, Fig.1A). By calculating the ventricles volumes, we found that MCC950(NLRP3 inflammasome specific inhibitor) treatment significantly reduced ventricles dilation at 3,7 , and 14 days after ICH-IVH (ICH-IVH vs MCC950, p<0.05, Fig.1B). In addition, ICH-IVH group started to occur obvious hydrocephalus at 3 days after ICH-IVH. The mNSS and corner test were used to assess neurofunction after ICH-IVH. Rats had severe neurodeficits after ICH-IVH and MCC950 treatment improved neurofunction at 3 and 7 days (Fig.1C and Fig.1D). As time goes on, inhibiting NLRP3 inflammasome gradually loss its function to improve neurodeficits according to mNSS and corner test results at 14 days after ICH-IVH (Fig. 1C and Fig.1D). Since the ventricle extension and neurodeficits occurred obviously on day 3 after ICH-IVH, we chose this time point (day 3 after ICH-IVH) to do further research. 


\section{NLRP3 Inflammasome was Activated after ICH-IVH in SVZ}

The expression of NLRP3 and NLRP3 inflammasome-related cytokines caspase-1 and IL-1 $\beta$ in SVZ were significantly increased on day 3 after ICH-IVH (sham vs ICH-IVH, p<0.01, Fig.2A-D), all of which were significantly decreased by MCC950 treatment (ICH-IVH vsMCC950, p<0.01, Fig.2A-D).

Immunofluorescence staining was performed to assess NLRP3 inflammasome related cytokines caspase- 1 and IL-1 $\beta$. What's more, both caspase- 1 and IL-1 $\beta$ positive cells in SVZ were obviously increased after $\mathrm{ICH}-\mathrm{IVH}$, and MCC950 treatment decreased this up-regulation (Fig.2E-G). Combined with neurobehavioral tests result, we demonstrated that NLRP3 inflammasome depended inflammation contributed to neurodeficits after hemorrhage.

\section{MCC950 Inhibited Microglia/macrophage Activation after ICH-IVH}

On day 3 after ICH-IVH, the immunofluorescence staining showed that NLRP3 was colocalized with microglia (Iba1) and macrophage (CD68) in SVZ (Fig.3A and Fig.3D). After ICH-IVH, microglia and macrophage were obviously activated, which was reflected by the increase of cells number (Fig.3B and Fig.3E). MCC950 treatment significantly inhibited the activation of microglia and macrophage (Fig.3B and Fig.3E). In addition, NLRP3 positive microglia/macrophage increased after ICH-IVH, and using inhibitor MCC950 decreased NLRP3 activation in microglia and macrophage (Fig.3C and Fig.3F). These results showed that NLRP3 activated in microglia/macrophage after ICH-IVH in SVZ, and NLRP3 related inflammation how to act to neurons and influence neurons function need to explore.

\section{Neurons Autophagy were Upregulated after ICH-IVH in SVZ}

According to previous suppose, we assessed autophagy in neurons. Autophagy was highly activated in neurons after ICH-IVH, the autophagy process specific markers LC3B and p62 were used to evaluated autophagy levels. Firstly, autophagy process was proved located in neurons after ICH-IVH. the autophagy process marker LC3B positive neurons increased after ICH-IVH, and MCC950 treatment decreased LC3B positive neurons in SVZ (Fig.4A and Fig.4C). The autophagosome degradation stage marker p62 also was observed in neurons which showed that p62 positive neurons decreased after ICH-IVH and MCC950 treatment inhibited this process (Fig.4B and Fig.4D). Then, the LC3B expression level increased and p62 expression level decreased in SVZ after ICH-IVH, using MCC950 to inhibit NLRP3 could reverse both LC3B and p62 expression levels (Fig.4E-H). These results indicated that neurons occurred severe autophagy after ICH-IVH, and inhibiting NLRP3 inflammasome reduced neurons autophagy.

\section{Excessive autophagy mediated neurons apoptosis after ICH-IVH in SVZ}

Excessive autophagy was observed in neurons after ICH-IVH. TUNEL staining was used to assess cells apoptosis. Compared with sham group, ICH-IVH group had more cells apoptosis in SVZ. MCC950 treatment decreased cells apoptosis (sham vs ICH-IVH, p<0.01; ICH-IVH vs MCC950, $p<0.05$, Fig.5A and Fig.5B). FJC staining was used to evaluate neuron condition. There were more damaged neurons (FJC positive cells) after ICH-IVH in SVZ, and MCC950 treatment reversed this trend (sham vs ICH-IVH, $p<0.01$; 
ICH-IVH vs MCC950, p<0.01, Fig.5C and Fig.5D). lavish autophagy mediated neuron apoptosis after ICHIVH, and NLRP3 inflammasome contributed to this process. Using MCC950 to inhibit NLRP3 activation could prevent neurons apoptosis, relieve the ventricular dilatation, and improve neruodeficits after ICHIVH.

\section{Effect of MCC950 on Transcriptional Phenotype after ICH-IVH in SVZ}

To explore the relationship between NLRP3 inflammasome and neuron autophagy, transcriptome sequencing was used. Compared with sham group, 565 genes expression changed ( 518 genes upregulated, 47 genes downregulated) (Fig.6A and Fig.6B). Heat-map of different expression genes after ICH-IVH contained NLRP3, autophagy, and apoptosis related genes (Fig.6C). According to GO enrichment, we found inflammatory response and innate immune response had obvious change (Fig.S1A). Focus on KEEG pathway enrichment, phagosome pathway changed drastically (36 genes expression changed in this pathway) (Fig.S1B). Compared with ICH-IVH group, MCC950 group had 167 genes expression changed (Fig.6D and Fig.6E), and heat-map of different expression genes after MCC950 treatment was used to select obviously related genes, which contained possible pathways that NLRP3 inflammasome mediated neuron autophagy through (Fig.6F). Combined with GO enrichment pathway and KEEG enrichment pathway, AMPK/Beclin-1 pathway was selected to further explore (Fig.S1C and Fig.S1D). The transcriptome sequencing results also supported our previous conclusion.

\section{NLRP3 upregulated autophagy through AMPK/Beclin-1 pathway}

After ICH-IVH, the expression of AMPK, p- AMPK, ULK1, and Beclin-1 were increased (sham vs ICH-IVH, p<0.01,Fig.7A-C, and Fig.7E-F). What's more, p- AMPK /AMPK was also increased (Fig.7D). Inhibiting NLRP3 inflammasome by MCC950 down-regulated the expression of key proteins in the AMPK/Beclin-1 pathway. Based on the above results, we speculated that NLRP3 inflammasome might mediate neuron apoptosis through AMPK/Beclin-1 pathway, and AMPK/Beclin-1 pathway also could regulate autophagy.

\section{IL-1 $\beta$ accelerated to excessive autophagy in PC12 cells}

IL-1 $\beta$ which could be secreted to extracellular from microglia/macrophage was the major cytokines after NLRP3 activated. Three dosages of IL-1 $\beta(0 \mu \mathrm{g} / \mathrm{L} ; 10 \mu \mathrm{g} / \mathrm{L} ; 20 \mu \mathrm{g} / \mathrm{L})$ was used to intervene PC12 cells which is a kind of neurons. Compared with vehicle group, there are more LC3B- and p62-positive neurons after IL-1 $\beta$ treated (Fig.8A and Fig.8B). the expression of LC3B and p62 indicated the autophagy level upregulated after IL-1 $\beta$ intervened and reached to peak at a dose of $10 \mu \mathrm{g} / \mathrm{L}$ (Fig.8C-E). However, IL-1 $\beta$ $(20 \mu \mathrm{g} / \mathrm{L})$ intervened PC12 cells, the autophagy related proteins had lower expression level than IL$1 \beta(10 \mu \mathrm{g} / \mathrm{L})$ group (Fig.8C-E). These results demonstrated IL-1 $\beta$ could upregulate autophagy in neurons as an important cytokine released after NLRP3 activated.

\section{IL-1 $\beta$ mediated neurons apoptosis in PC12 cells}

To explore whether IL-1 $\beta$ treatment influenced neurons function, we evaluated the apoptosis of neurons. Compared with vehicle group, the apoptosis related protein cleaved-caspase3 had higher expression level, 
and increased along with the dose of IL-1 $\beta$ (Fig.9A and Fig.9D). The results of TUNEL staining and apoptosis assay showed the same conclusion (Fig.9B-C and Fig.9E). The results indicated that IL-1 $\beta$ leaded to neurons apoptosis by upregulating the autophagy level.

\section{Discussion}

In this study, we found that activation of NLRP3 in microglia/macrophage after ICH-IVH aggravated neurons apoptosis by upregulating autophagy through AMPK/Beclin-1 pathway in SVZ. Besides, IL-1 $\beta$ whose secretion was mainly promoted by NLRP3 activation mediated autophagy-induced PC12 cells apoptosis (Fig. 10). This study provide compelling evidence that NLRP3-mediated neurons apoptosis played an important role in the pathogenesis of $\mathrm{ICH}-\mathrm{IVH}$.

After ICH, resident glial cells were activated and circulating immune cells were recruited to participate in the occurrence and development of neuroinflammation[26-28]. Microglia was the resident macrophage distributed in brain that could be rapidly activated to mediate neuroinflammation in response to pathological conditions, including hypoxia, infection, and brain tissue injury[29]. Besides directly attack to neurons, the polarized microglia could also damage the neurons indirectly by changing the microenvironment via releasing neurotoxicity factors such as IL-1 $\beta$ or recruiting other neurotoxicity cells such as macrophages to amplify inflammatory response[30]. Inflammation cytokines(IL-1 $\beta$, IL-6, IL-27, and TNF-a) mainly released from microglia/macrophage and neutrophils during neuroinflammation aggravated secondary injury to neurons[14,31,32]. Thus, inhibiting neuroinflammation might play a protective role in $\mathrm{ICH}$.

Nucleotide-binding oligomerization domain-like receptor containing pyrin domain 3(NLRP3) inflammasome, the apoptosis-associated speck-like protein, has been proposed as a crucial mediator in innate immunity[33]. The activated NLRP3 could cleave pro-IL-1 $\beta$ and pro-IL-18 into their mature and functional form, resulting in the activation of subsequent inflammatory response[33, 34]. Recent studies indicated NLRP3 inflammasome could also polarize microglia and exacerbate ischemia/hemorrhageinduced brain injury $[35,36]$. NLRP3 activation aggravate neuronal cell death and behavioral deficits, Knockdown or downregulating NLRP3 could improve neurological functions of ICH animals[37]. In this study, we found that NLRP3 was widely activated in microglia/macrophage on day 3 after ICH-IVH, while the neuroinflammation was supposed to reach to the peak at the same time. In addition, we found that using NLRP3 inhibitor MCC950 could improve cognitive and motor function of ICH rats. Individual neuronal cells and their synapses constituted neuronal circuits control all kinds of neurofunction. Furthermore, to explore how the NLRP3 activation in microglia/macrophage influences cognitive and motor function after hemorrhage, we aim to neurons.

As the terminally differentiated cells, neurons did not divide and replicate themselves, which was the main reason why a severe injury in brain was often difficult to recover[38]. Autophagy was a conserved intracellular process to degrade dysfunctional organelles and protein aggregates, and played an important role in maintaining neuronal homeostasis[38]. Much of the evidence to support this derives 
from studies adjusting autophagy in neurons and observing neurodegeneration, especially in $A D$ and $\mathrm{PD}$ [39-41]. Normal autophagy was considered as a protective factor against neurodegeneration, infection, and brain injury disease[42], however, autophagy disfunction was associated with increased susceptibility of neurons to ischemic injury. It was reported that the activation of autophagy was coincide with axonal swelling of PC12 cells when nerve growth factor was deprived or cells were in excitotoxicity, suggesting a close relationship between autophagic process and neurite degeneration[43]. In addition, neurotoxin exposure induced apoptosis with a concomitant increase of autophagy flux in primary cortical neurons[44]. All of these evidences indicated that autophagy flux participated in neuronal injury in many kinds of CNS diseases. However, the role of autophagy after ICH still remained controversy. Studies found that autophagy-related disorders promoted the occurrence of stroke in some cases[45], and autophagy exacerbated brain injury after $\mathrm{ICH}$. Autophagic cell death of neurons after $\mathrm{ICH}$ was confirmed by using conditional knockout Atg7 mice[46]. Although autophagy was involved in promoting brain injury during the acute phase of $\mathrm{ICH}$, studies showed that autophagy had a neuroprotective function via clearing up the accumulation of cell rubbish [47], and the anti-neuronal apoptosis effects was related to the enhancement of autophagy[48]. Several studies showed that ICH induced autophagy of immune cells, especially microglia/macrophage, contributing to improvement of outcomes by regulating inflammation [49-51]. Hence, we aimed to explore the relationship between neuronal apoptosis and autophagy in acute phase of $\mathrm{ICH}$, and to prove that excessive neuronal autophagy aggravated neuronal apoptosis, which contributed to neurodeficits after ICH-IVH.

NLRP3 activation in microglia/macrophage was the key process to adjust neuronal autophagy, and according to the RNA sequencing results, we found that NLRP3 mediated neuronal autophagy through AMPK/Beclin-1 pathway. Since NLRP3 activation could promote the release of cytokines, among which IL-1 $\beta$ played the most important role among these factors[52, 53], we further explored the role of IL-1 $\beta$, and found that IL-1 $\beta$ was the key factor mediating excessive autophagy and neuronal apoptosis after NLRP3 activation by using PC12 cells in vitro.

Several limitations need to be mentioned in this study. First, NLRP3 plays multifunctional roles in adaptive immunity, and NLRP3 inflammasome is activated in many kinds of neural cells. Further research is needed to investigate the other mechanisms underlying the neuroprotective effects of NLRP3 in secondary brain injury after ICH-IVH. Second, since NLRP3 is not only expressed on microglia/macrophage as our immunofluorescence staining showed, further study about the roles of NLRP3 on other CNS cells, such as astrocytes, is necessary. Third, how the neuronal autophagy affects neuronal function after hemorrhage and the related mechanism associated with NLRP3 should be more deeply evaluated. Recent studies and our present study have demonstrated the effectiveness of inhibiting NLRP3 expression by using MCC950[54, 55]. However, the time window and stability of MCC950 are very limited. This study only focused on the early pathophysiological changes (3 days) in SVZ after ICH-IVH, the longer time research is needed in the further study. At the same time, the effects of NLRP3 on specific cell types require more precise gene-editing techniques, such as the use of cre/loxp rat to edit NLRP3 genes in specific cells. 
In sum, we found that NLRP3 was activated to promote the release of IL-1 $\beta$ to extracellular from microglia/macrophage after ICH-IVH, which mediated neuronal apoptosis by upregulating autophagy through AMPK/Beclin-1 pathway. These results provide a potential therapeutic target to ICH-IVH patients in acute stage.

\section{Conclusions}

In conclusion, we demonstrated that NLRP3 inflammasome activation in microglia/macrophage attenuated neuroinflammation and neuronal apoptosis after ICH-IVH, which was mediated by upregulating autophagy through AMPK/Beclin-1 signaling pathway in neurons. At least in part, NLRP3 related extracellular cytokine IL-1 $\beta$ contributed to this process. Therefore, NLRP3 activation may be a potential therapeutic strategy in the management of ICH-IVH patients.

\section{Abbreviations}

$A D$ : Alzheimer disease

AMPK: Adenosine 5'-monophosphate (AMP)-activated protein kinase

FJC: Fluoro-Jade C

Iba1: Ionized calciumbinding adaptor molecule 1

$\mathrm{ICH}$ : Intracerebral hemorrhage

ICH-IVH: Intracerebral hemorrhage with ventricle extension

IL-1 $\beta$ : Interleukin-1 $\beta$

IL-18: Interleukin-18

IL-6: Interleukin-6

IL-27: Interleukin-27

LC3B: Microtubule-associated proteins 1A/1B light chain 3B

mNSS: The modified neurological severity score

MRI: Magnetic resonance imaging

NLRP3: NACHT, LRR and PYD domains-containing protein 3

p-AMPK: Phospho- Adenosine 5'-monophosphate (AMP)-activated protein kinase 
PBS: Phosphate-buffered saline

PD: Parkinson disease

p62: Sequestosome-1

SVZ: Subventricular zone

TNF-a: Tumor necrosis factor-a

Tunel: Terminal deoxynucleotidyl transferase dUTP nick end labeling

ULK1: Serine/threonine-protein kinase ULK1

\section{Declarations}

Ethics approval and consent to participate:

All institutional and national guidelines for the care and use of laboratory animals were followed.

Consent for publication: Not applicable

Availability of data and materials: Not applicable

Competing interests:

All authors declare that they have no conflicts of interest.

Funding:

This work was supported by the Talents Training Program of Army Medical University No. 2019MPRC021 and Brain Collaborative Innovation Center of ChongQing No. 4174D7.

\section{Author's contributions:}

HF and TNC made substantial contributions to the conception and design. ZQZ performed the experiments and acquired the data. PWG and ZCJ statistic and analysis the related data. All authors read and approved the final manuscript.

Acknowledgements: Not applicable

\section{References}

1. Ziai WC, Carhuapoma JR: Intracerebral Hemorrhage.Continuum (Minneap Minn) 2018, 24:16031622. 
2. Hanley DF: Intraventricular hemorrhage: severity factor and treatment target in spontaneous intracerebral hemorrhage.Stroke 2009, 40:1533-1538.

3. Mayer SA, Rincon F: Treatment of intracerebral haemorrhage.Lancet Neurol 2005, 4:662-672.

4. Stein M, Luecke M, Preuss M, Boeker DK, Joedicke A, Oertel MF: Spontaneous intracerebral hemorrhage with ventricular extension and the grading of obstructive hydrocephalus: the prediction of outcome of a special life-threatening entity.Neurosurgery 2010, 67:1243-1251; discussion 1252.

5. Pappas A, Adams-Chapman I, Shankaran S, McDonald SA, Stoll BJ, Laptook AR, Carlo WA, Van Meurs KP, Hintz SR, Carlson MD, et al: Neurodevelopmental and Behavioral Outcomes in Extremely Premature Neonates With Ventriculomegaly in the Absence of Periventricular-Intraventricular Hemorrhage.JAMA Pediatr 2018, 172:32-42.

6. Bu Y, Chen M, Gao T, Wang X, Li X, Gao F: Mechanisms of hydrocephalus after intraventricular haemorrhage in adults.Stroke Vasc Neurol 2016, 1:23-27.

7. Ziai WC: Hematology and inflammatory signaling of intracerebral hemorrhage.Stroke 2013, 44:S7478.

8. Wang T, Nowrangi D, Yu L, Lu T, Tang J, Han B, Ding Y, Fu F, Zhang JH: Activation of dopamine D1 receptor decreased NLRP3-mediated inflammation in intracerebral hemorrhage mice.J Neuroinflammation 2018, 15:2.

9. Zhou Y, Wang Y, Wang J, Anne Stetler R, Yang QW: Inflammation in intracerebral hemorrhage: from mechanisms to clinical translation.Prog Neurobio/ 2014, 115:25-44.

10. Pan Y, Chen XY, Zhang QY, Kong LD: Microglial NLRP3 inflammasome activation mediates IL-1betarelated inflammation in prefrontal cortex of depressive rats.Brain Behav Immun 2014, 41:90-100.

11. Scheiblich H, Schlutter A, Golenbock DT, Latz E, Martinez-Martinez P, Heneka MT: Activation of the NLRP3 inflammasome in microglia: the role of ceramide.J Neurochem 2017, 143:534-550.

12. Cordero MD, Williams MR, Ryffel B: AMP-Activated Protein Kinase Regulation of the NLRP3 Inflammasome during Aging. Trends Endocrinol Metab 2018, 29:8-17.

13. Li J, Chen J, Mo H, Chen J, Qian C, Yan F, Gu C, Hu Q, Wang L, Chen G: Minocycline Protects Against NLRP3 Inflammasome-Induced Inflammation and P53-Associated Apoptosis in Early Brain Injury After Subarachnoid Hemorrhage.Mol Neurobio/ 2016, 53:2668-2678.

14. Ren H, Kong Y, Liu Z, Zang D, Yang X, Wood K, Li M, Liu Q: Selective NLRP3 (Pyrin Domain-Containing Protein 3) Inflammasome Inhibitor Reduces Brain Injury After Intracerebral Hemorrhage.Stroke 2018, 49:184-192.

15. Galluzzi L, Bravo-San Pedro JM, Blomgren K, Kroemer G: Autophagy in acute brain injury.Nat Rev Neurosci 2016, 17:467-484.

16. Cho MH, Cho K, Kang HJ, Jeon EY, Kim HS, Kwon HJ, Kim HM, Kim DH, Yoon SY: Autophagy in microglia degrades extracellular beta-amyloid fibrils and regulates the NLRP3 inflammasome.Autophagy 2014, 10:1761-1775. 
17. Houtman J, Freitag K, Gimber N, Schmoranzer J, Heppner FL, Jendrach M: Beclin1-driven autophagy modulates the inflammatory response of microglia via NLRP3.EMBO J 2019, 38.

18. You T, Cheng Y, Zhong J, Bi B, Zeng B, Zheng W, Wang H, Xu J: Roflupram, a Phosphodiesterase 4 Inhibitior, Suppresses Inflammasome Activation through Autophagy in Microglial Cells.ACS Chem Neurosci 2017, 8:2381-2392.

19. Qin Y, Qiu J, Wang P, Liu J, Zhao Y, Jiang F, Lou H: Impaired autophagy in microglia aggravates dopaminergic neurodegeneration by regulating NLRP3 inflammasome activation in experimental models of Parkinson's disease.Brain Behav Immun 2020.

20. Uddin MS, Stachowiak A, Mamun AA, Tzvetkov NT, Takeda S, Atanasov AG, Bergantin LB, AbdelDaim MM, Stankiewicz AM: Autophagy and Alzheimer's Disease: From Molecular Mechanisms to Therapeutic Implications. Front Aging Neurosci 2018, 10:04.

21. Mizushima N, Levine B, Cuervo AM, Klionsky DJ: Autophagy fights disease through cellular selfdigestion.Nature 2008, 451:1069-1075.

22. Zhang X, Wei M, Fan J, Yan W, Zha X, Song H, Wan R, Yin Y, Wang W: Ischemia-induced upregulation of autophagy preludes dysfunctional lysosomal storage and associated synaptic impairments in neurons.Autophagy 2020:1-24.

23. Chen Q, Zhang J, Guo J, Tang J, Tao Y, Li L, Feng H, Chen Z: Chronic hydrocephalus and perihematomal tissue injury developed in a rat model of intracerebral hemorrhage with ventricular extension. Trans/ Stroke Res 2015, 6:125-132.

24. Chen J, Sanberg PR, Li Y, Wang L, Lu M, Willing AE, Sanchez-Ramos J, Chopp M: Intravenous administration of human umbilical cord blood reduces behavioral deficits after stroke in rats.Stroke 2001, 32:2682-2688.

25. Okauchi M, Hua Y, Keep RF, Morgenstern LB, Xi G: Effects of deferoxamine on intracerebral hemorrhage-induced brain injury in aged rats.Stroke 2009, 40:1858-1863.

26. Dong Y, Fan C, Hu W, Jiang S, Ma Z, Yan X, Deng C, Di S, Xin Z, Wu G, et al: Melatonin attenuated early brain injury induced by subarachnoid hemorrhage via regulating NLRP3 inflammasome and apoptosis signaling.J Pineal Res 2016, 60:253-262.

27. Khey KMW, Huard A, Mahmoud SH: Inflammatory Pathways Following Subarachnoid Hemorrhage.Cell Mol Neurobiol 2020, 40:675-693.

28. Xu W, Li T, Gao L, Zheng J, Yan J, Zhang J, Shao A: Apelin-13/APJ system attenuates early brain injury via suppression of endoplasmic reticulum stress-associated TXNIP/NLRP3 inflammasome activation and oxidative stress in a AMPK-dependent manner after subarachnoid hemorrhage in rats.J Neuroinflammation 2019, 16:247.

29. Gris T, Laplante P, Thebault P, Cayrol R, Najjar A, Joannette-Pilon B, Brillant-Marquis F, Magro E, English SW, Lapointe R, et al: Innate immunity activation in the early brain injury period following subarachnoid hemorrhage.J Neuroinflammation 2019, 16:253.

30. Wang X, Sun G, Feng T, Zhang J, Huang X, Wang T, Xie Z, Chu X, Yang J, Wang H, et al: Sodium oligomannate therapeutically remodels gut microbiota and suppresses gut bacterial amino acids- 
shaped neuroinflammation to inhibit Alzheimer's disease progression.Cell Res 2019, 29:787-803.

31. Wang M, Ye X, Hu J, Zhao Q, Lv B, Ma W, Wang W, Yin H, Hao Q, Zhou C, et al: NOD1/RIP2 signalling enhances the microglia-driven inflammatory response and undergoes crosstalk with inflammatory cytokines to exacerbate brain damage following intracerebral haemorrhage in mice.J Neuroinflammation 2020, 17:364.

32. Zhao X, Ting SM, Liu CH, Sun G, Kruzel M, Roy-O'Reilly M, Aronowski J: Neutrophil polarization by IL27 as a therapeutic target for intracerebral hemorrhage.Nat Commun 2017, 8:602.

33. Bracey NA, Duff HJ, Muruve DA: Hierarchical regulation of wound healing by NOD-like receptors in cardiovascular disease.Antioxid Redox Signal 2015, 22:1176-1187.

34. Martinez GJ, Robertson S, Barraclough J, Xia Q, Mallat Z, Bursill C, Celermajer DS, Patel S: Colchicine Acutely Suppresses Local Cardiac Production of Inflammatory Cytokines in Patients With an Acute Coronary Syndrome.J Am Heart Assoc 2015, 4:e002128.

35. Ma Q, Chen S, Hu Q, Feng H, Zhang JH, Tang J: NLRP3 inflammasome contributes to inflammation after intracerebral hemorrhage.Ann Neurol 2014, 75:209-219.

36. Yang Z, Zhong L, Xian R, Yuan B: MicroRNA-223 regulates inflammation and brain injury via feedback to NLRP3 inflammasome after intracerebral hemorrhage.Mol Immunol 2015, 65:267-276.

37. Xiao L, Zheng H, Li J, Wang Q, Sun H: Neuroinflammation Mediated by NLRP3 Inflammasome After Intracerebral Hemorrhage and Potential Therapeutic Targets.Mol Neurobiol 2020, 57:5130-5149.

38. Stavoe AKH, Holzbaur ELF: Autophagy in Neurons.Annu Rev Cell Dev Biol 2019, 35:477-500.

39. Ahmed I, Liang Y, Schools S, Dawson VL, Dawson TM, Savitt JM: Development and characterization of a new Parkinson's disease model resulting from impaired autophagy.J Neurosci 2012, 32:1650316509.

40. Hara T, Nakamura K, Matsui M, Yamamoto A, Nakahara Y, Suzuki-Migishima R, Yokoyama M, Mishima K, Saito I, Okano H, Mizushima N: Suppression of basal autophagy in neural cells causes neurodegenerative disease in mice.Nature 2006, 441:885-889.

41. Sato S, Uchihara T, Fukuda T, Noda S, Kondo H, Saiki S, Komatsu M, Uchiyama Y, Tanaka K, Hattori $\mathrm{N}$ : Loss of autophagy in dopaminergic neurons causes Lewy pathology and motor dysfunction in aged mice.Sci Rep 2018, 8:2813.

42. Menzies FM, Fleming A, Caricasole A, Bento CF, Andrews SP, Ashkenazi A, Fullgrabe J, Jackson A, Jimenez Sanchez M, Karabiyik C, et al: Autophagy and Neurodegeneration: Pathogenic Mechanisms and Therapeutic Opportunities.Neuron 2017, 93:1015-1034.

43. Yang $Y, X u$ K, Koike T, Zheng X: Transport of autophagosomes in neurites of PC12 cells during serum deprivation.Autophagy 2008, 4:243-245.

44. Li S, Jiang Z, Chai W, Xu Y, Wang Y: Autophagy activation alleviates nonylphenol-induced apoptosis in cultured cortical neurons.Neurochem Int 2019, 122:73-84.

45. Feldt-Rasmussen U: Fabry disease and early stroke.Stroke Res Treat 2011, 2011:615218. 
46. Chen CW, Chen TY, Tsai KL, Lin CL, Yokoyama KK, Lee WS, Chiueh CC, Hsu C: Inhibition of autophagy as a therapeutic strategy of iron-induced brain injury after hemorrhage.Autophagy 2012, 8:15101520.

47. Duan XC, Wang W, Feng DX, Yin J, Zuo G, Chen DD, Chen ZQ, Li HY, Wang Z, Chen G: Roles of autophagy and endoplasmic reticulum stress in intracerebral hemorrhage-induced secondary brain injury in rats.CNS Neurosci Ther 2017, 23:554-566.

48. Chen J, Wang L, Wu C, Hu Q, Gu C, Yan F, Li J, Yan W, Chen G: Melatonin-enhanced autophagy protects against neural apoptosis via a mitochondrial pathway in early brain injury following a subarachnoid hemorrhage.J Pineal Res 2014, 56:12-19.

49. Shi H, Wang J, Wang J, Huang Z, Yang Z: IL-17A induces autophagy and promotes microglial neuroinflammation through ATG5 and ATG7 in intracerebral hemorrhage.J Neuroimmuno/ 2018, 323:143-151.

50. Yang Z, Liu B, Zhong L, Shen H, Lin C, Lin L, Zhang N, Yuan B: Toll-like receptor-4-mediated autophagy contributes to microglial activation and inflammatory injury in mouse models of intracerebral haemorrhage.Neuropathol App/ Neurobiol 2015, 41:e95-106.

51. Yu A, Zhang T, Zhong W, Duan H, Wang S, Ye P, Wang J, Zhong S, Yang Z: miRNA-144 induces microglial autophagy and inflammation following intracerebral hemorrhage.Immunol Lett 2017, 182:18-23.

52. Mangan MSJ, Olhava EJ, Roush WR, Seidel HM, Glick GD, Latz E: Targeting the NLRP3 inflammasome in inflammatory diseases.Nat Rev Drug Discov 2018, 17:588-606.

53. Swanson KV, Deng M, Ting JP: The NLRP3 inflammasome: molecular activation and regulation to therapeutics.Nat Rev Immunol 2019, 19:477-489.

54. Wu D, Chen Y, Sun Y, Gao Q, Li H, Yang Z, Wang Y, Jiang X, Yu B: Target of MCC950 in Inhibition of NLRP3 Inflammasome Activation: a Literature Review.Inflammation 2020, 43:17-23.

55. Yang $Y$, Wang $H$, Kouadir $M$, Song $H$, Shi F: Recent advances in the mechanisms of NLRP3 inflammasome activation and its inhibitors. Cell Death Dis 2019, 10:128.

\section{Figures}


A

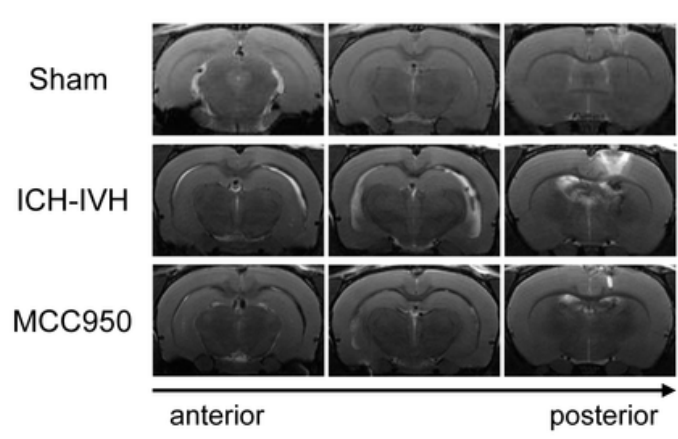

Day 7

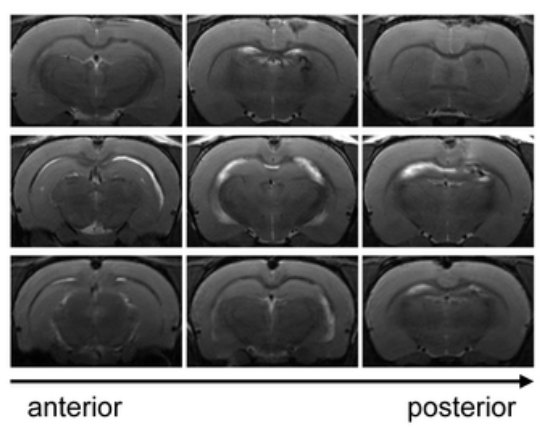

Day 14

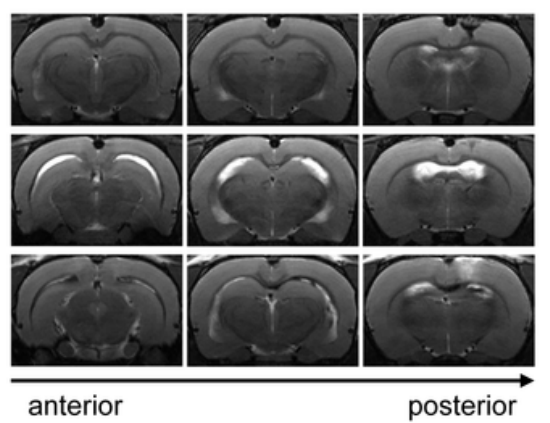

B

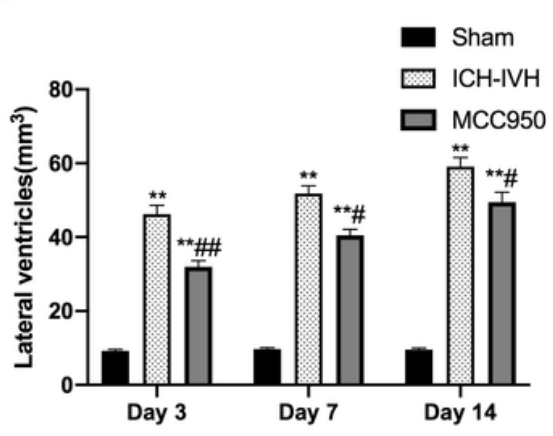

C

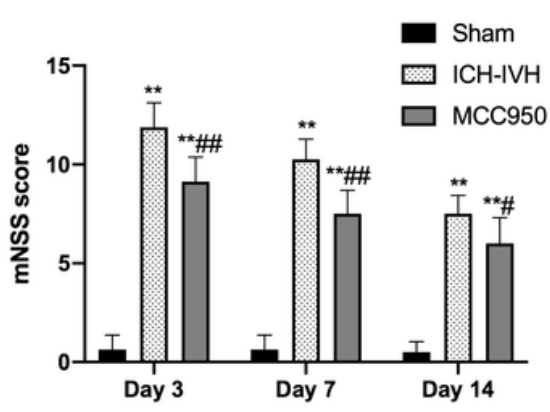

D

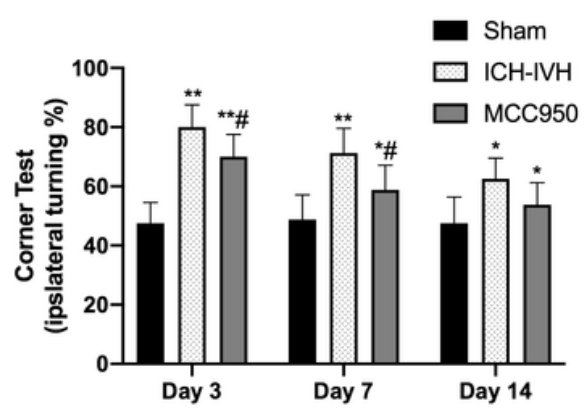

\section{Figure 1}

Hydrocephalus and neurodeficits after intracerebral hemorrhage with ventricles extension (IC-IVH). (A, B) Representative T2WI imaging sections at 3, 7, and 14 days with normal saline or MCC950 treated after ICH-IVH (A), lateral ventricles volumes calculated by T2WI images (B). (C, D) Neurological tests were performed to evaluate the motor, sensory, and balance function in rats after receiving saline or MCC950, mNSS was used to evaluate neurofunction comprehensively (C), Corner tests aimed at motor function after hemorrhage (D). ( $A$ and $B, n=5 /$ group; $C$ and $D, n=12 /$ group). Results are presented as mean $\pm S D$, ${ }^{*} \mathrm{P}<0.01$ and ${ }^{*} \mathrm{P}<0.05$ versus Sham group, \#\#P $<0.01$ and $\# \mathrm{P}<0.05 \mathrm{ICH}-\mathrm{IVH}$ group versus MCC950 group. 

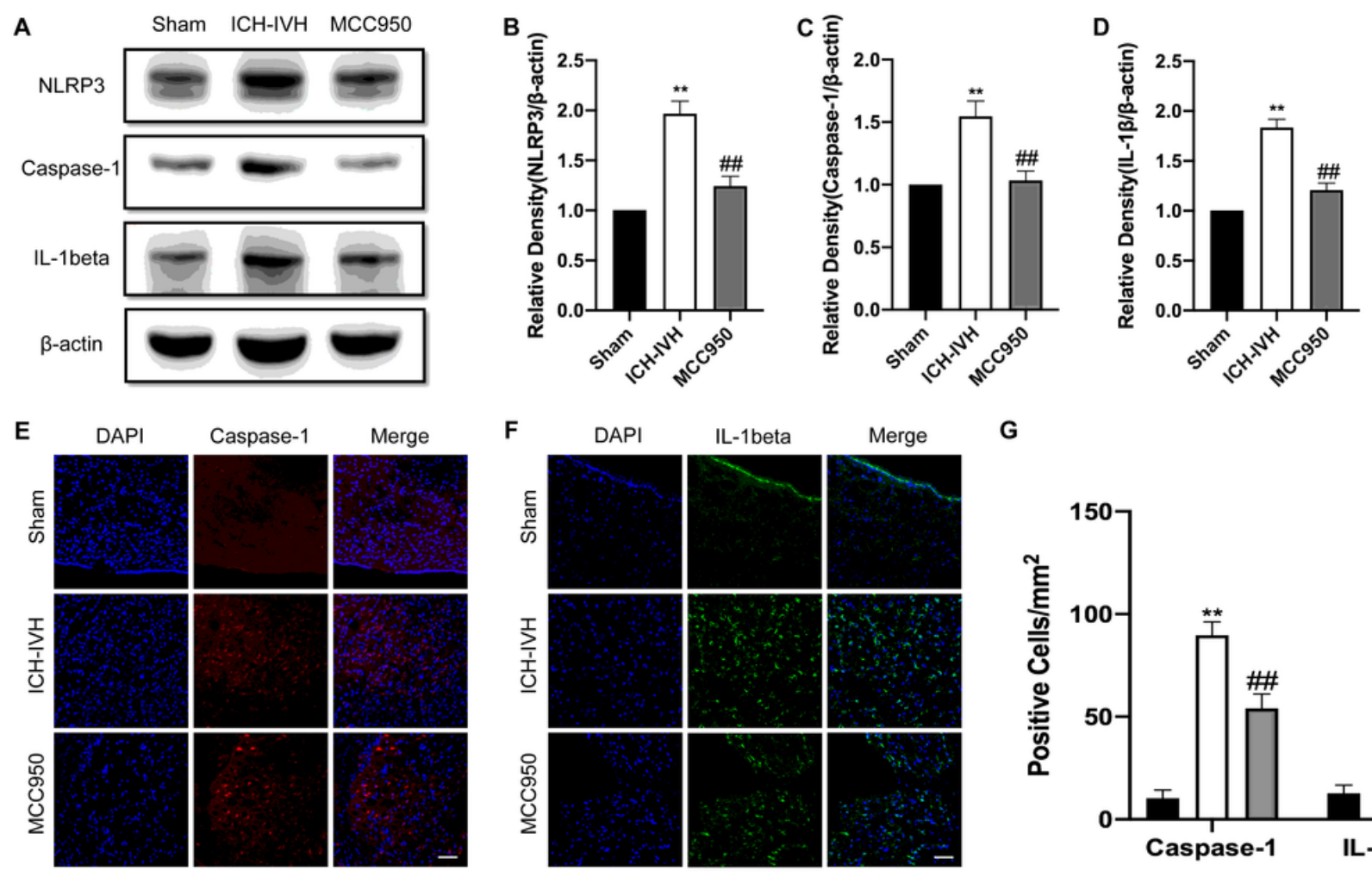

G

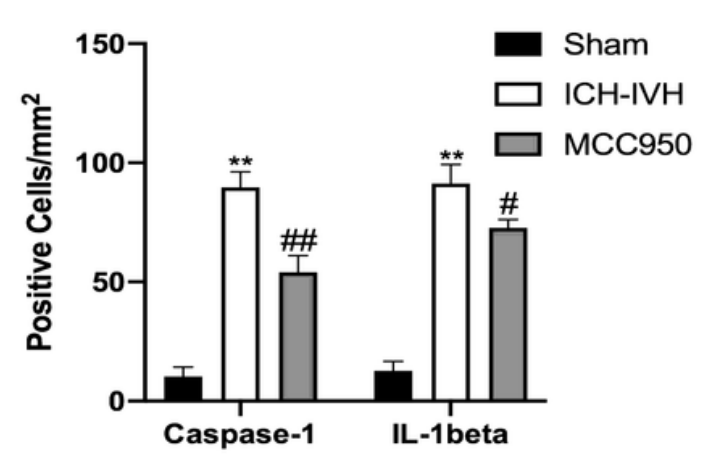

Figure 2

NLRP3 inflammasome activated and MCC950 decreased neuro-inflammasome after ICH-IVH in SVZ. (A) Representative western blots showed the expression of NLRP3, Caspase- 1 , and IL-1 $\beta$ under MCC950 treatment after ICH-IVH. (B to D) The analysis results of NLRP3, caspase-1, and IL-1 $\beta$ after ICH-IVH in SVZ. (E, F) Representative immunofluorescence staining images of Caspase- 1 and IL-1 $\beta$ positive cells in SVZ. Bar $=50 \mu \mathrm{m}$. (G) Statistical results of Caspase- 1 and IL-1 $\beta$ positive cells in SVZ. (A, $n=6 /$ group; $E$ and $F, n=6$ /group). Results showed with mean $\pm S D$, ${ }^{\star} * P<0.01$ and ${ }^{*} P<0.05$ versus Sham group, \#\#P<0.01 and \#P $<0.05 \mathrm{ICH}-\mathrm{IVH}$ group versus MCC950 group. 
A

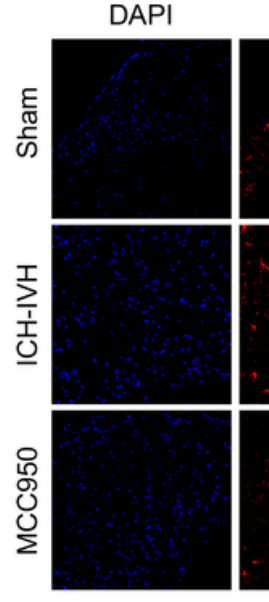

D

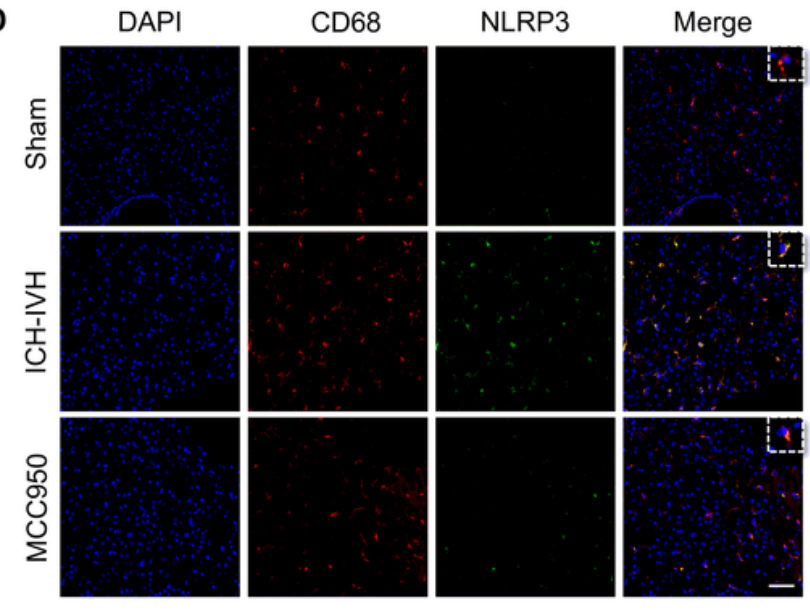

B

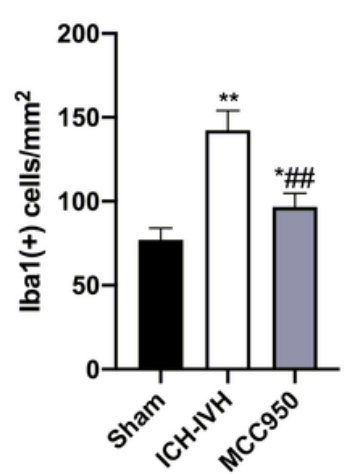

E

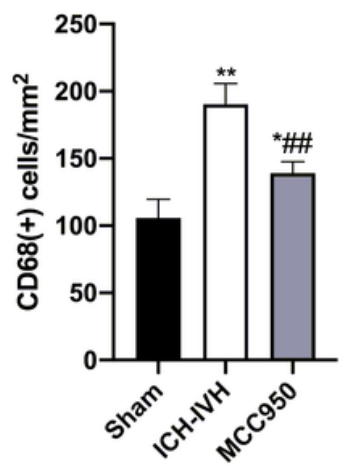

C

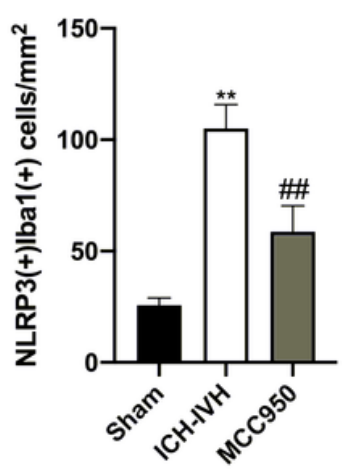

F

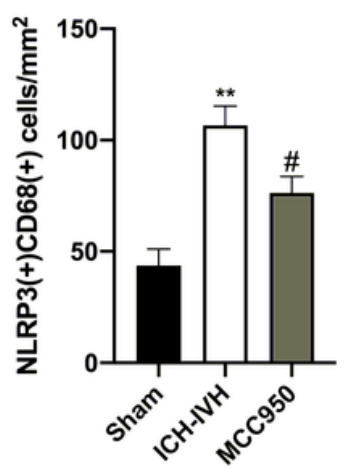

Figure 3

NLRP3 inflammasome located in activated microglia/macrophage after ICH-IVH. (A) representative photos of double immunofluorescence staining of NLRP3 expressed at lba1 positive cells in SVZ. (D) immunofluorescence staining images of CD68 and NLRP3 positive cells in SVZ. (B and E) statistic results of Iba1 or CD68 positive cells in SVZ after ICH-IVH and MCC950 treatment. (C and F) statistic results of NLRP3 positive microglia/macrophage in SVZ. (A and D, $n=6 /$ group). Bar=50 $\mu$ m. Results are presented as mean $\pm S D$, **P $<0.01$ versus Sham group, \#\#P $<0.01$ and \#P $<0.05$ ICH-IVH group versus MCC950 group. 
A

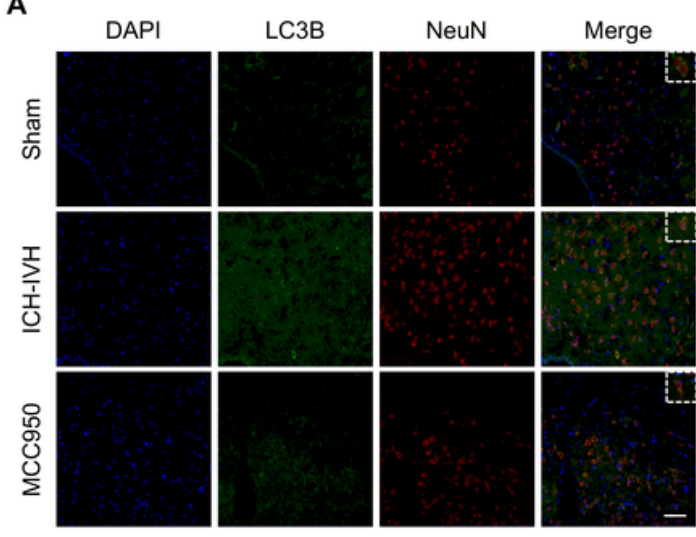

B

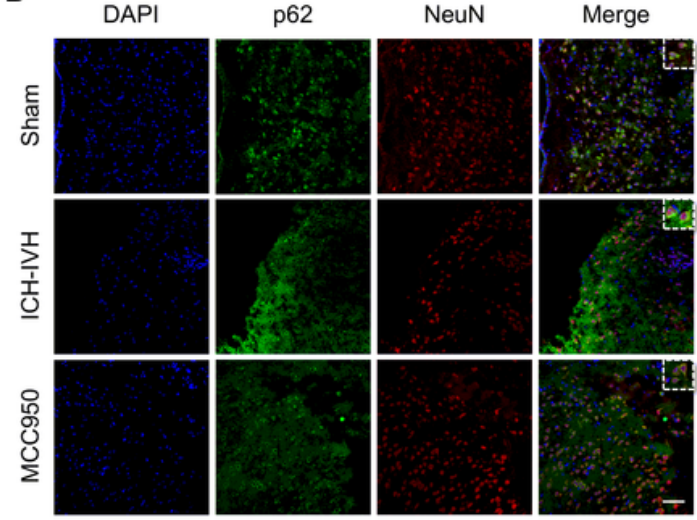

C

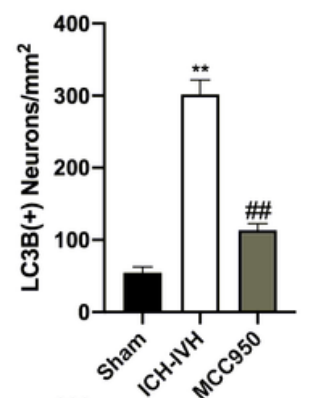

D

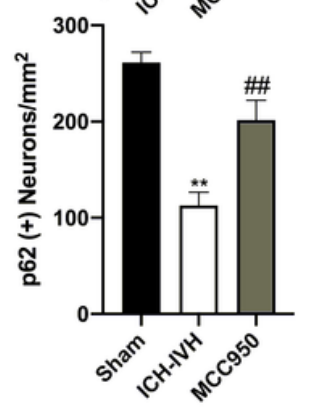

E

Sham ICH-IVH MCC950

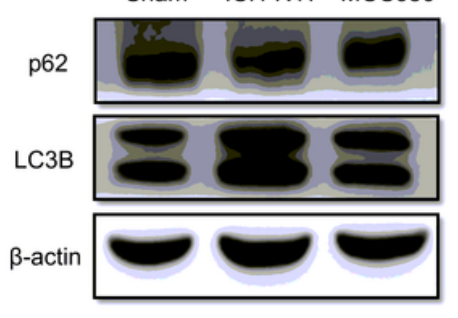

$\mathbf{F}$

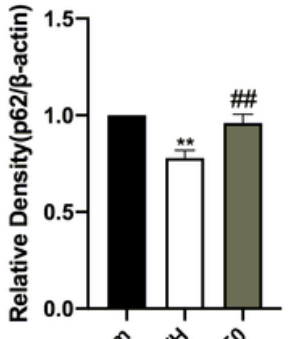

$\mathbf{G}$

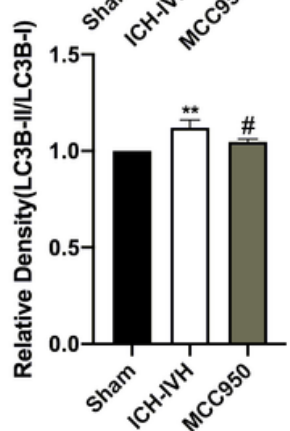

H

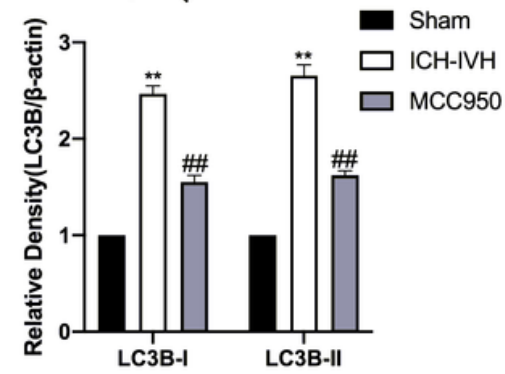

Figure 4

Autophagy upregulated in neurons located in SVZ after ICH-IVH. (A) Representative immunofluorescence staining images of LC3 positive neurons in SVZ. (B) Representative immunofluorescence staining images of p62 positive neurons in SVZ. (C, D) statistic results of LC3 and p62 positive neurons with normal saline and MCC950 in SVZ. (E) representative western blots images of the expression of LC3 and p62 after ICHIVH. (F to H) the analysis results of LC3B-II:LC3B-I ratio, LC3, and p62 in different group. (A and B, $\mathrm{n}=6$ /group; $\mathrm{E}, \mathrm{n}=6$ /group). Bar $=50 \mu \mathrm{m}$. Results are presented as mean $\pm S D, * * P<0.01$ versus Sham group, \#\#P $<0.01$ and \#P $<0.05 \mathrm{ICH}-\mathrm{IVH}$ group versus MCC950 group. 


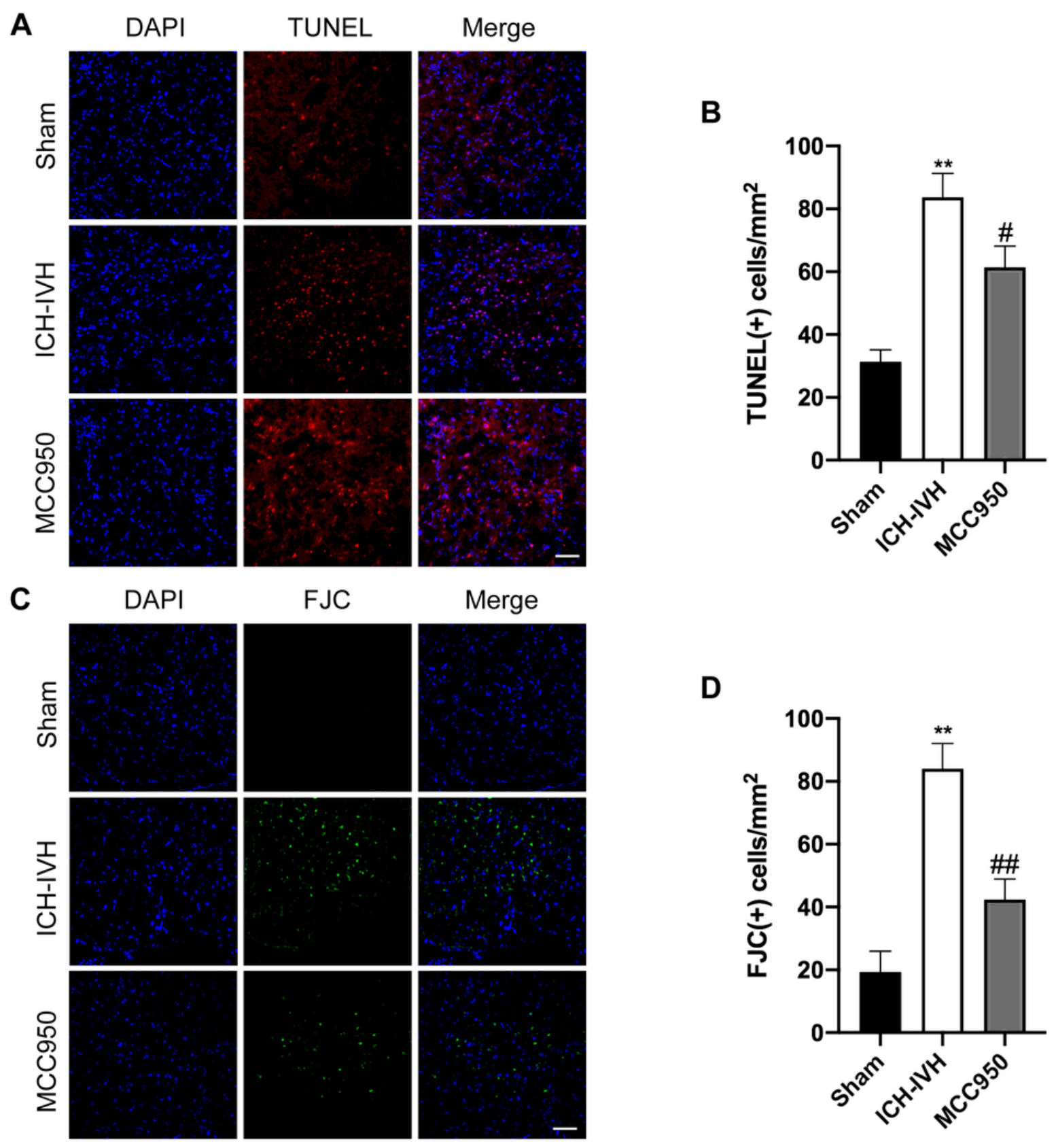

Figure 5

Neurons degenerated and apoptosis after ICH-IVH in SVZ. (A and B) representative immunofluorescence staining images of TUNEL cells in SVZ (A), and analysis results of TUNEL cells of different groups in SVZ (B). (C and D) immunofluorescence staining images showed FJC (+) neurons in SVZ, and statistic results

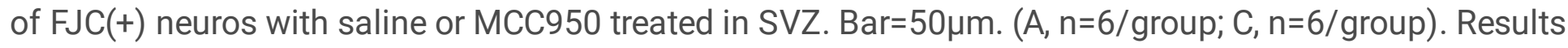
are presented as mean $\pm S D$, $* * P<0.01$ versus Sham group, \#\#P $<0.01$ and \#P $<0.05 \mathrm{ICH}-\mathrm{IVH}$ group versus MCC950 group. 
A

Sham versus $\mathrm{ICH}-\mathrm{IVH}$

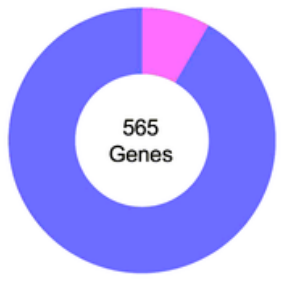

- $8.32 \%$ Upregulated

- $91.68 \%$ Downregulated

D

MCC950 versus ICH-IVH

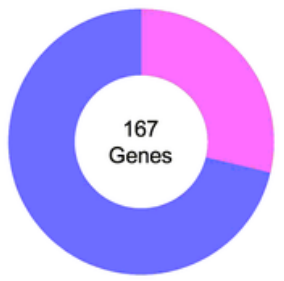

28.74\% Upregulated

- $71.26 \%$ Downregulated
B

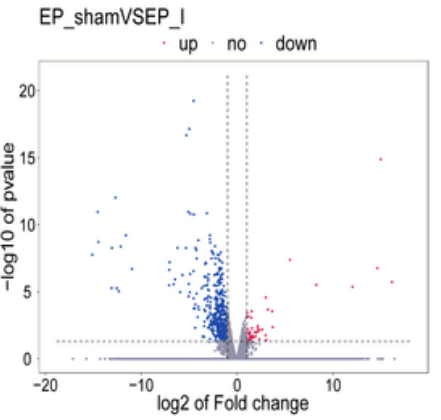

E

EP_MCCVSEP_।

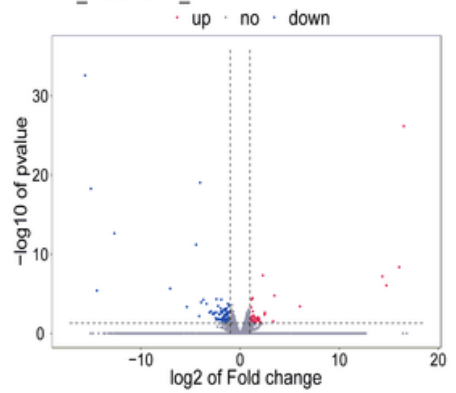

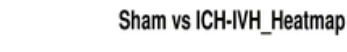

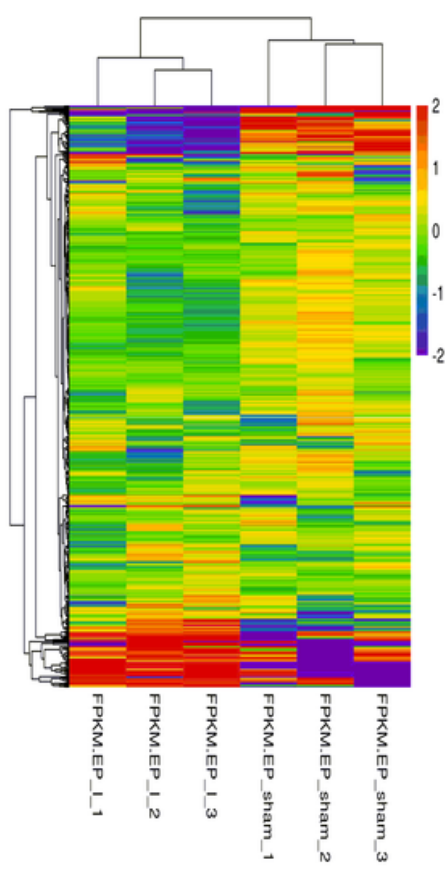

MCC950 vs ICH-IVH_Heatmap

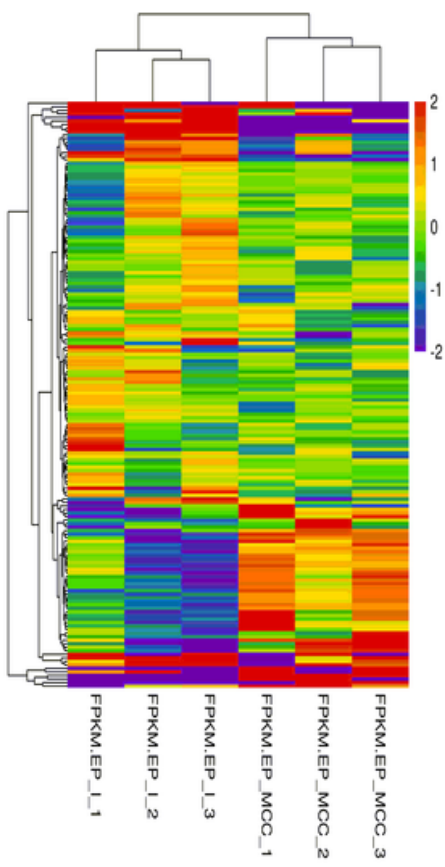

Figure 6

Transcriptional analysis of SVZ tissues identified AMPK/ULK1/Beclin-1 as potential mediator of the NLRP3 inflammasome and neuron autophagy after ICH-IVH. (A and D) different expression genes (upregulated and downregulated) in sham group, ICH-IVH group, and MCC950 treatment group. (B and E) Volcano plot showed differentially expressed genes in SVZ on day 3 after ICH-IVH (B); volcano plot after ICH-IVH compared with MCC950 treatment (E). ( $\mathrm{C}$ and F) heatmap of the Z score of genes identified by PCA for each sample. Data were clustered hierarchically in GENE-E, and colored according to row minimum and maximum. 

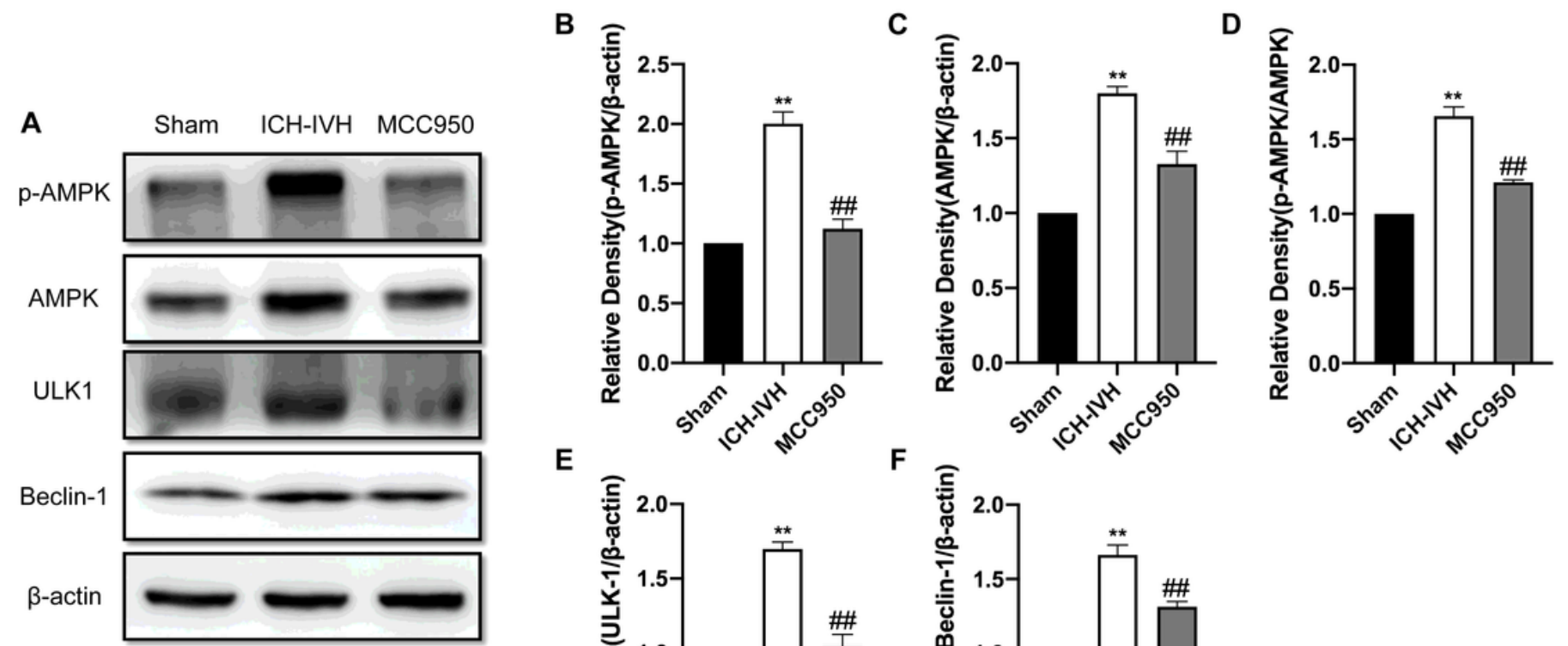

\section{Figure 7}

Autophagy related pathway AMPK/ULK1/Beclin-1 upregulated after ICH-IVH in SVZ. representative western blots images of AMPK/ULK1/Beclin-1 pathway (A), and quantitative analyses of AMPK phosphorylation, and the expression of AMPK, ULK1, and Beclin-1 (B to F). ( $n=6 /$ group). Data were represented as mean $\pm S D$, ${ }^{\star *} \mathrm{P}<0.01$ versus Sham group, \#\#P $<0.01 \mathrm{ICH}-\mathrm{IVH}$ group versus MCC950 group. 


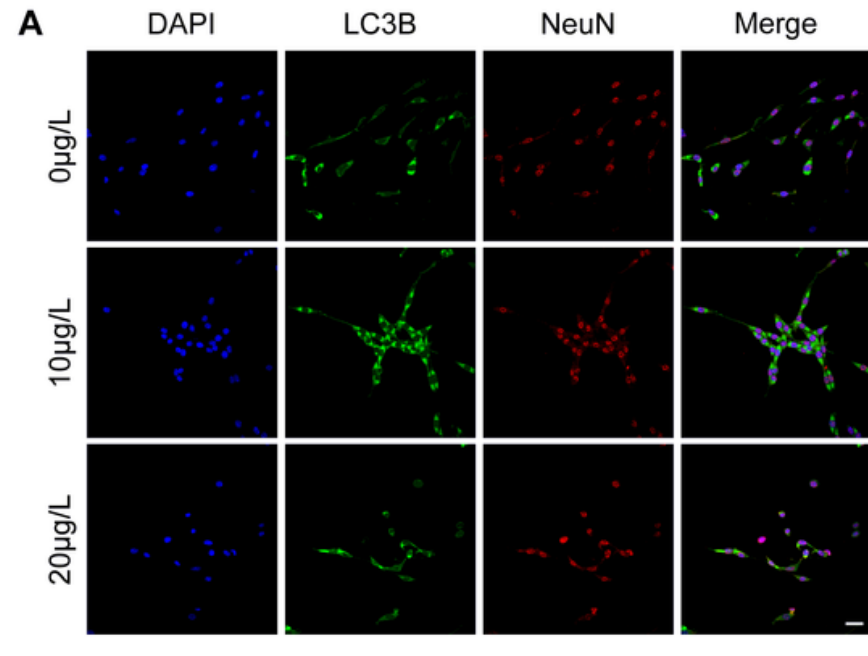

C

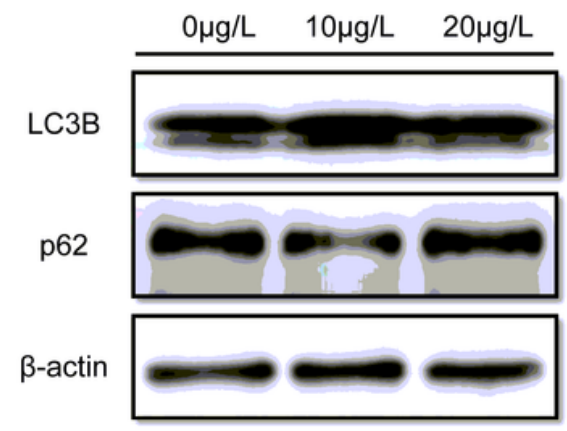

B

DAPI
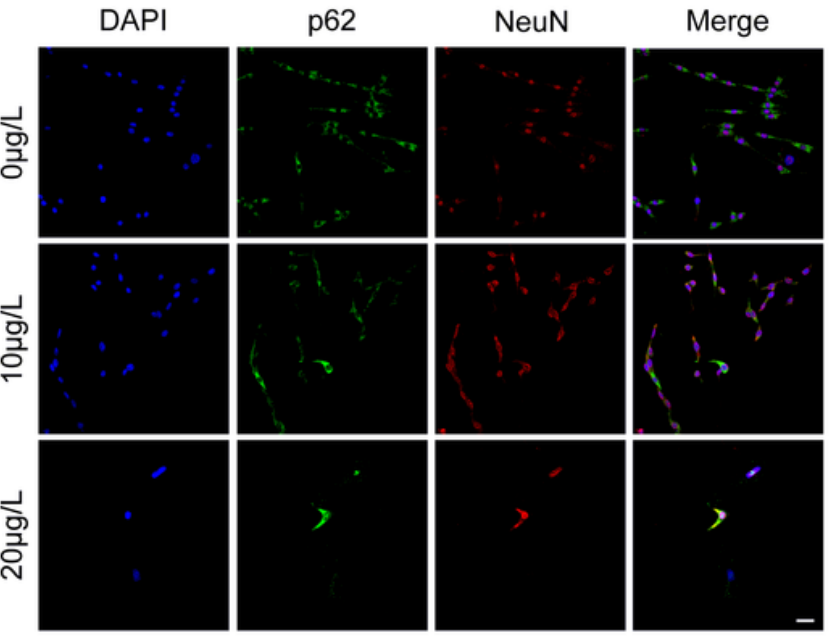

E

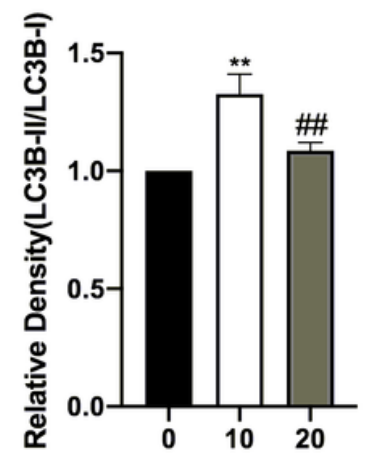

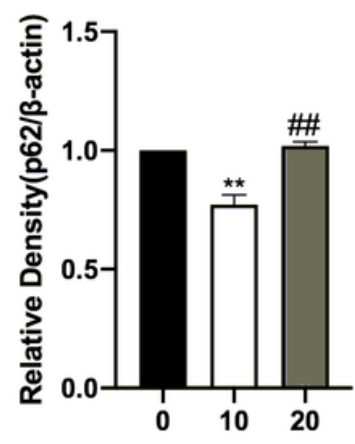

\section{Figure 8}

Different concentrations IL-1 $\beta$ mediated autophagy flux changed in PC12 cells. (A and B) Representative immunofluorescence staining images of LC3B- and p62- positive PC12 cells. Bar=50 $\mu \mathrm{m}$. ( $n=6 /$ group). (C) representative western blots images of LC3B and p62 expressions in PC12 cells. (D and E) statistic results of LC3B-II:LC3B-I ratio and p62 after different dosages IL-1 $\beta$ treated. ( $n=6 /$ group). Data were represented as mean $\pm S D$, ${ }^{\star *} \mathrm{P}<0.01$ versus Sham group, $\# \# \mathrm{P}<0.0110 \mu \mathrm{g} / \mathrm{L}$ group versus $20 \mu \mathrm{g} / \mathrm{L}$ group. 

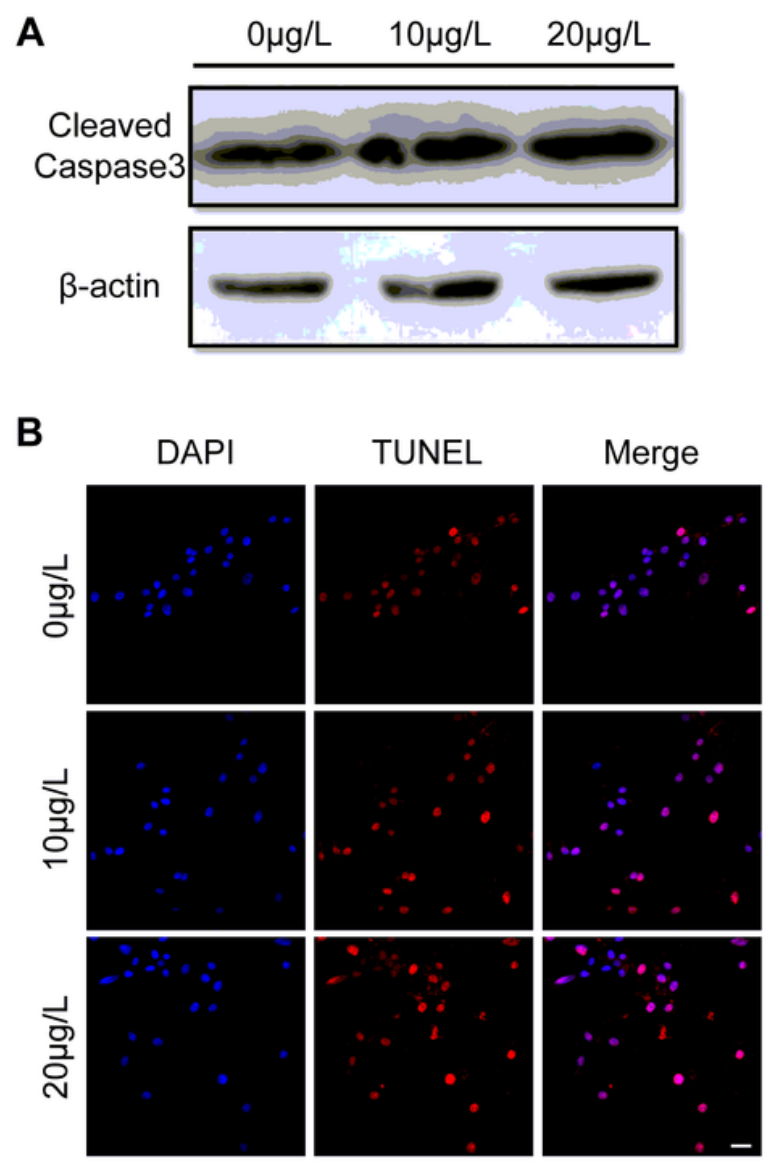

C
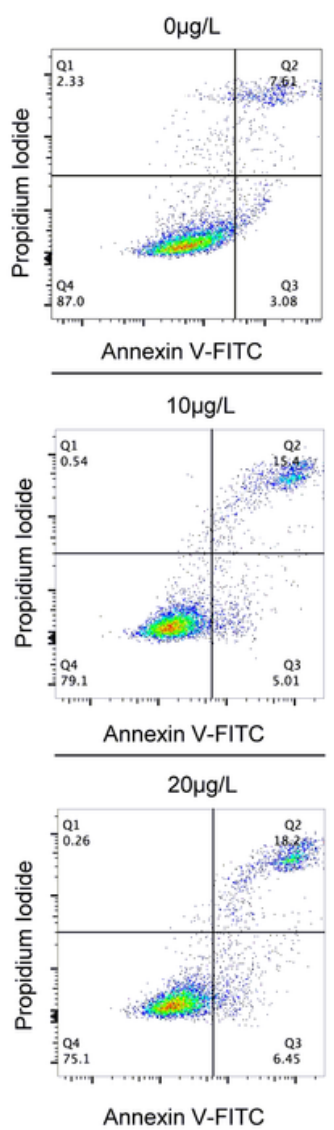

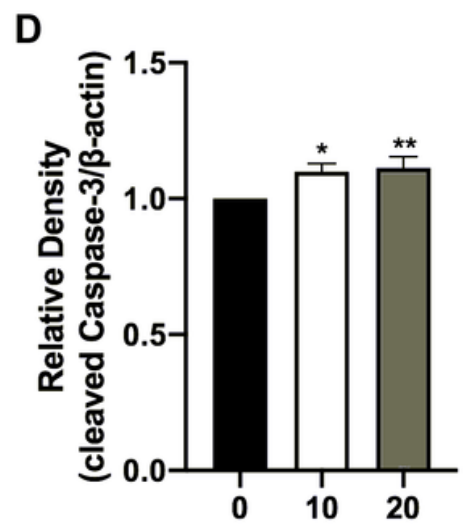

E

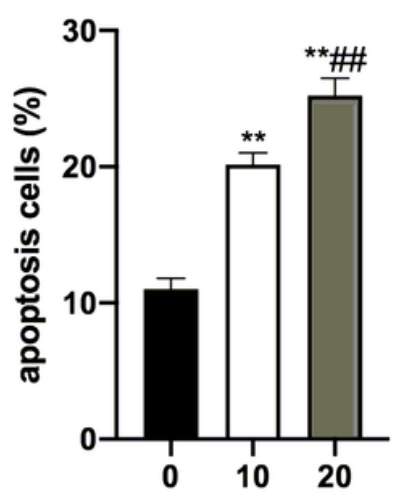

Figure 9

Virous dosages IL-1 $\beta$ mediated apoptosis in PC12 cells. (A and D) Representative western blots images of Cleaved-caspase3 after IL-1 $\beta$ treated(A), and analysis result of Cleaved-caspase3 expression in PC12 cells(D). (B and E) representative immunofluorescence staining images of TUNEL cells(B), and statistic result of TUNEL cells after virous dosages IL-1 $\beta$ treated(E). Bar=50 $\mu \mathrm{m}$. ( $n=6 /$ group). (C) representative images of flow cytometry showed early/late apoptotic cells after different dosages IL-1 $\beta$ treated. $(n=6 /$ group $)$. Data were represented as mean $\pm S D$, $* * P<0.01$ and $* P<0.05$ versus Sham group, $\# \# P<$ $0.0110 \mu \mathrm{g} / \mathrm{L}$ group versus $20 \mu \mathrm{g} / \mathrm{L}$ group. 


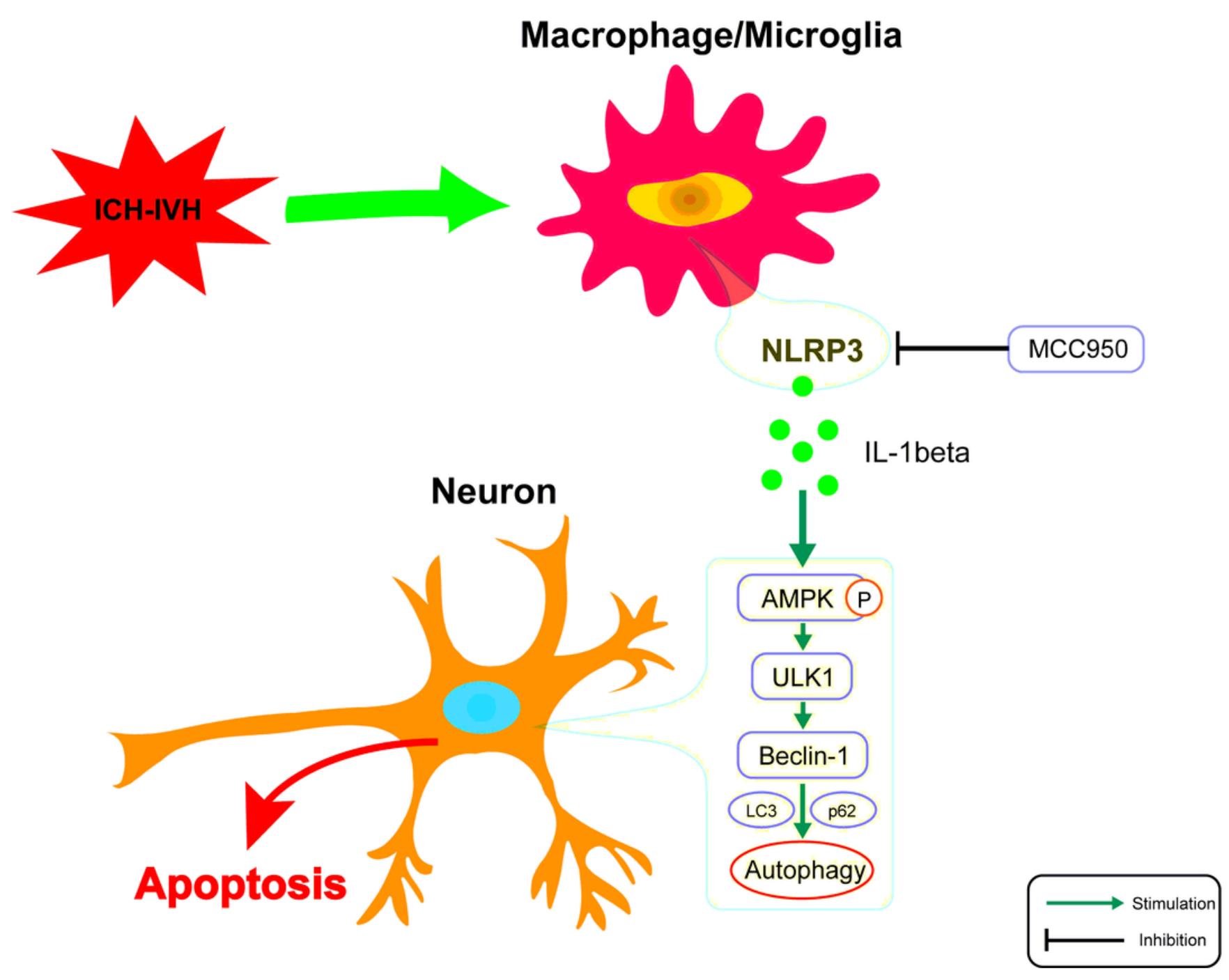

Figure 10

Schematic mechanism of microglia/macrophage-derived NLRP3 inflammasome ICH-IVH induced neuroinflammasome. ICH-IVH activated microglia/macrophage with NLRP3 inflammasome. Upon activated, IL$1 \beta$ was released which upregulated autophagy through AMPK/Beclin-1 pathway in neurons. Excessive autophagy mediated neurons apoptosis and neurodeficits. NLRP3 inhibitor MCC950 could attenuate ICHIVH induced inflammasome injury.

\section{Supplementary Files}

This is a list of supplementary files associated with this preprint. Click to download.

- Figure.S1.tif 